\title{
Fracture functions in different kinematic regions and their factorizations
}

\author{
Kai-bao Chen ${ }^{* \dagger}$ \\ Institute of Theoretical Physics, Chinese Academy of Sciences, P.O. Box 2735, Beijing 100190, \\ China \\ School of Physical Sciences, University of Chinese Academy of Sciences, Beijing 100049, China \\ E-mail: chenkbditp.ac.cn
}

\begin{abstract}
Fracture functions are parton distributions of a hadron in the presence of an almost collinear particle observed in the final state. They are important ingredients in QCD factorization for processes where a particle is produced diffractively. We take the production of a Drell-Yan lepton pair and a diffractively produced photon in hadron collisions to discuss this. Through explicit one-loop calculations, we show that both the transverse momentum dependent and the integrated fracture functions can be factorized with twist-2 parton distribution functions and fragmentation functions in different kinematic regions. The complete perturbative coefficients are obtained. Evolution equations for these fracture functions are also derived.
\end{abstract}

XXVII International Workshop on Deep-Inelastic Scattering and Related Subjects - DIS2019

8-12 April, 2019

Torino, Italy

\footnotetext{
*Speaker.

${ }^{\dagger}$ Supported by China Postdoctoral Science Foundation (No.2018M631588).
} 


\section{Introduction}

In semi-inclusive high energy reactions, the observed particles can come from the fragmentation of a scattered parton, however, they can also be found among the remnants of the struck target, or the so called target fragmentation region. E.g., the semi-inclusive deeply inelastic scattering (SIDIS) in target fragmentation region, or diffractive Drell-Yan lepton pair and hadron production at forward rapidities. If the transverse momentum of the produced particle is large, it is well known that QCD collinear factorization works, and the cross section can be predicted in terms of parton distribution functions (PDFs) and/or fragmentation functions (FFs). However, if the transverse momentum is small, this factorization fails due to the large logs in perturbative coefficients.

It has been proposed in [U] to use fracture functions to describe particle productions in target fragmentation region of SIDIS. In this talk, we discuss the factorizations for the production of a Drell-Yan lepton pair associated with a diffractively produced photon in hadron-hadron collisions. The cross-section or the hadronic tensor can be factorized in terms of integrated fracture functions when the transverse momentum $q_{\perp}$ of the lepton pair is large. When $q_{\perp} \sim \Lambda_{Q C D}$, the factorization can be done in terms of the transverse momentum dependent (TMD) fracture functions. By explicit one-loop calculations, we show in momentum space and the Fourier transformed impact parameter $b$-space that these fracture functions can be further factorized in terms of PDFs and FFs when the transverse momentum is large or $b$ is small. Evolution equations for the fracture functions are also derived. The main results can be found in [[]].

\section{Factorization of the cross-section}

We consider the process of the production of a Drell-Yan lepton pair associated with a diffractively produced photon in hadron-hadron collisions as shown in Fig.W(a), i.e.,

$$
h_{A}\left(P_{A}\right)+h_{B}\left(P_{B}\right) \rightarrow \gamma^{*}(q)+\gamma(k)+X
$$

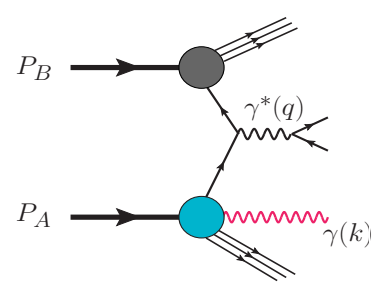

(a)

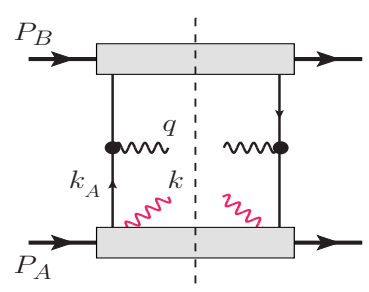

(b)

Figure 1: Feynman diagrams for the process and the hadronic tensor.

In the center of mass frame of $h_{A}$ and $h_{B}$, the momenta of the virtual and real photon are:

$$
q^{\mu}=\left(q^{+}, q^{-}, \vec{q}_{\perp}\right), \quad k^{\mu}=\left(k^{+}, k^{-}, \vec{k}_{\perp}\right) \sim Q\left(1, \lambda^{2}, \lambda, \lambda\right),
$$

where $Q=\sqrt{q^{2}} \gg \Lambda_{Q C D}$ is the invariant mass of the lepton pair. The real photon is almost collinear with $h_{A}$, i.e., $\lambda \ll 1$. The hadronic tensor is denoted in Fig.W(b), and it is defined as

$$
W^{\mu v}=\sum_{X} \int \frac{d^{4} x}{(2 \pi)^{4}} e^{i q \cdot x}\left\langle h_{A}, h_{B}\left|\bar{q}(0) \gamma^{v} q(0)\right| X, \gamma(k)\right\rangle\left\langle\gamma(k), X\left|\bar{q}(x) \gamma^{\mu} q(x)\right| h_{A}, h_{B}\right\rangle .
$$


This hadronic tensor will take different factorization forms in different regions.

Factorization with TMD fracture function. When $q_{\perp} \sim \lambda Q$, at the leading power, the hadronic tensor can be factorized with the TMD fracture function, the TMD PDF, and a soft factor, i.e.,

$$
\begin{array}{r}
W^{\mu v}=-g_{\perp}^{\mu v} \frac{1}{N_{c}} H\left(Q, \zeta_{u}^{2}, \zeta_{v}^{2}\right) \int d^{2} k_{A \perp} d^{2} k_{B_{\perp}} d^{2} \ell_{\perp} \delta^{2}\left(k_{A \perp}+k_{B \perp}+\ell_{\perp}-q_{\perp}\right) \\
\cdot \tilde{S}\left(\ell_{\perp}, \rho^{2}\right) f_{\bar{q}}\left(y, k_{B \perp}, \zeta_{v}\right) \mathscr{F}_{q}\left(x, k_{A \perp}, \xi, k_{\perp}, \zeta_{u}\right)+\cdots
\end{array}
$$

the TMD fracture function is defined as

$$
\mathscr{F}_{q}\left(x, k_{A \perp}, \xi, k_{\perp}\right)=\int \frac{d z^{-} d^{2} z_{\perp}}{2(2 \pi)^{3}} e^{i \tilde{k}_{A} \cdot \hat{z}} \sum_{X}\left\langle h_{A}\left|\bar{q}(0) \mathscr{L}_{u}(0) \gamma^{+}\right| X, \gamma(k)\right\rangle\left\langle\gamma(k), X\left|\mathscr{L}_{u}^{\dagger}(\hat{z}) q(\hat{z})\right| h_{A}\right\rangle
$$

with $\tilde{k}_{A}^{\mu}=\left(x P_{A}^{+}, 0, k_{A \perp}^{1}, k_{A \perp}^{2}\right), \hat{z}^{\mu}=\left(0, z^{-}, z_{\perp}\right)$ and $\xi=k^{+} / P_{A}^{+}$. We regularize the light-cone singularity by putting the gauge link off the light-cone direction, i.e., $u^{\mu}=\left(u^{+}, u^{-}, 0,0\right), u^{+} \ll u^{-}$. The TMD fracture function will then have dependence on the parameter $\zeta_{u}^{2}=\left(2 u \cdot P_{A}\right)^{2} / u^{2}$.

Factorization with integrated fracture function. When $q_{\perp} \gg \lambda Q$, the standard collinear factorization applies. The hadronic tensor is factorized with the integrated fracture function and the collinear PDF

$$
W^{\mu v}=\int d x_{A} d x_{B} \sum_{a, b} w_{a b}^{\mu v}\left(x_{A} P_{A}, x_{B} P_{B}, q\right) F_{a}\left(x_{A}, \xi, k_{\perp}\right) f_{b / B}\left(x_{B}\right)
$$

where $w_{a b}^{\mu v}$ is the perturbative coefficient. The integrated fracture function is defined as

$$
F_{q}\left(x, \xi, k_{\perp}\right)=\int \frac{d \lambda}{4 \pi} e^{i x P_{A}^{+} \lambda} \sum_{X}\left\langle h_{A}\left|\bar{q}(0) \mathscr{L}_{n}(0) \gamma^{+}\right| X, \gamma(k)\right\rangle\left\langle\gamma(k), X\left|\mathscr{L}_{n}^{\dagger}(\lambda n) q(\lambda n)\right| h_{A}\right\rangle
$$

\section{Factorization of the fracture functions}

In general, fracture function is a nonperturbative object. However, when the transverse momentum of the observed particle is relatively large, there are effects which are calculable with perturbative QCD, which means we can factorized them further. We show these factorizations for the TMD and integrated fracture functions respectively in the following.

\subsection{Factorization of the TMD fracture function at large transverse momentum}

In the case $k_{A \perp}, k_{\perp} \gg \Lambda_{Q C D}$ and also $\ell_{\perp}=k_{A \perp}+k_{\perp} \gg \Lambda_{Q C D}$, only real diagrams in Fig.D and [ $]$ (conjugate diagrams not shown) with large transverse momentum parton emission will contribute. The factorization is expected to take the following form:

$$
\mathscr{F}_{q}\left(x, k_{A \perp}, \xi, k_{\perp}\right)=\int_{x+\xi}^{1} \frac{d y}{y}\left[f_{q}(y) \mathscr{C}_{q}\left(x / y, \xi / y, k_{A \perp}, k_{\perp}\right)+f_{g}(y) \mathscr{C}_{g}\left(x / y, \xi / y, k_{A \perp}, k_{\perp}\right)\right],
$$



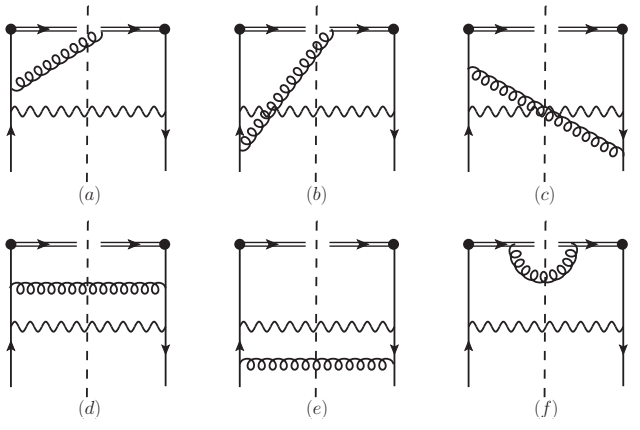

Figure 2: Real diagrams for quark initial states.

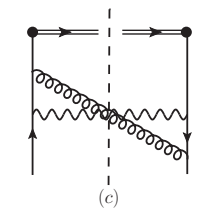

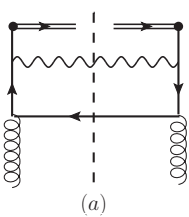
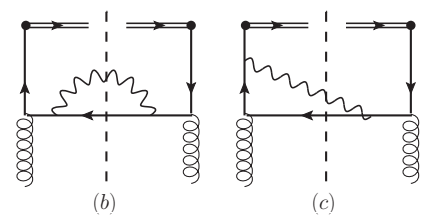

Figure 3: Real diagrams for gluon initial states.

where $f_{q, g}(y)$ are twist-2 PDFs. The hard coefficients $\mathscr{C}_{q, g}$ are calculated straightforwardly:

$$
\begin{aligned}
& \mathscr{C}_{q}\left(x, \xi, k_{A \perp}, k_{\perp}\right)=\frac{\alpha_{s} C_{F} e^{2}}{\pi^{2} k_{\perp}^{2}} {\left[\frac{\delta(z) \gamma(\xi)}{\ell_{\perp}^{2}}\left(\ln \frac{\zeta_{u}^{2} \bar{\xi}^{2}}{\ell_{\perp}^{2}}-1\right)+\frac{\gamma(\xi) / z_{+}-x-\bar{\xi}}{\ell_{\perp}^{2}}-\frac{x\left(\xi^{2} \ell_{\perp}^{2}+z^{2} k_{\perp}^{4} / \ell_{\perp}^{2}\right)}{\xi \bar{\xi}^{2} D_{d}^{2}}\right.} \\
&\left.+\frac{\gamma(\xi) \bar{\xi} / z_{+}-x-1}{\bar{\xi} D_{d}}+\frac{k_{\perp}^{2}\left(\gamma(\xi)-z^{3}+3 z^{2}+\xi z-4 z\right)}{\xi \bar{\xi} \ell_{\perp}^{2} D_{d}}\right]+\mathscr{O}(\varepsilon), \quad \text { (3.2) } \\
& \mathscr{C}_{g}\left(x, \xi, k_{A \perp}, k_{\perp}\right)=\frac{\alpha_{s} e^{2} z}{2 \pi^{2}}\left[\frac{x z k_{\perp}^{2}-2 \xi^{2} x D_{c}}{\xi^{2} \bar{\xi}^{2} D_{c} D_{d}^{2}}+\frac{2 x^{2}(1+z)-\xi^{2} x-3 x+1}{\xi^{2} \bar{\xi} D_{c} D_{d}}+\frac{z\left(\bar{\xi}^{2}-2 x z\right)}{\xi^{2} D_{c} \ell_{\perp}^{2}}\right. \\
&\left.-\frac{2 x^{2}(z-\xi)-\xi^{3}-3 \xi^{2} x-x}{\xi \bar{\xi} D_{d} \ell_{\perp}^{2}}-\frac{x z k_{\perp}^{2}}{\xi \bar{\xi}^{2} D_{d}^{2} \ell_{\perp}^{2}}\right]+\mathscr{O}(\varepsilon),
\end{aligned}
$$

in which we have used the notations: $z=1-x-\xi, \bar{\xi}=1-\xi, \gamma(\xi)=1+\bar{\xi}^{2}, D_{c}=\left(\ell_{\perp}+z k_{\perp} / \xi\right)^{2}$, $D_{d}=\left(\ell_{\perp}-z k_{\perp} / \bar{\xi}\right)^{2}+x z k_{\perp}^{2} / \xi \bar{\xi}^{2}$.

\subsection{Factorization of the TMD fracture function in impact parameter space}

When we transform the fracture function into the impact parameter $b$-space, i.e.,

$$
\hat{F}_{q}\left(x, b, \xi, k_{\perp}\right)=\int d^{2-\varepsilon} \ell_{\perp} e^{-i b \cdot \ell_{\perp}} \mathscr{F}_{q}\left(x, \ell_{\perp}-k_{\perp}, \xi, k_{\perp}\right)
$$

there is another factorization relating the fracture function to PDFs and FFs when the impact parameter $b$ is small. The factorization reads:

$$
\hat{F}_{q}\left(x, b, \xi, k_{\perp}\right)=\sum_{a, b} \int \frac{d y}{y} f_{a}(y)\left[\hat{\mathscr{H}}_{a}\left(\frac{x}{y}, \frac{\xi}{y}, b, k_{\perp}\right)+\int \frac{d z}{z} D_{b}(z) \hat{\mathscr{H}}_{a b}\left(x, \xi, z, b, k_{\perp}\right)\right]+\mathscr{O}\left(b^{2}\right) .
$$

At one loop, besides the real diagrams in Fig. [ and B, we also have virtual diagrams as in Fig.T. The explicit results for each diagram can be found in []. We emphasize here that Fig.L(a), (b) and Fig. If(c) all have $1 / \varepsilon_{c}^{2}$ double poles which come from the overlap of soft and collinear pole. However, they correctly cancel each other, and the final results contain only a collinear single pole. The divergent part for the quark initial state in Fig.D and $⿴ 囗 十$ can be factorized with the quark 


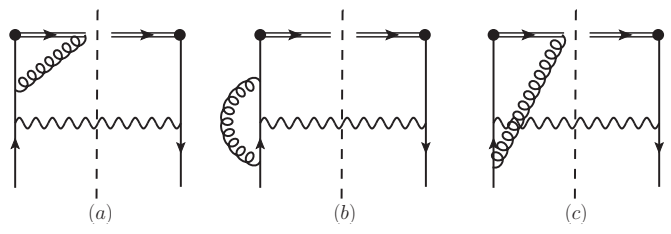

Figure 4: Virtual diagrams for the quark fracture function.

distribution function. The perturbative coefficient function is extracted as:

$$
\begin{aligned}
\hat{\mathscr{C}}_{q}^{(1)} & \left(x, \xi, b, k_{\perp}\right)=\frac{e^{2} \alpha_{s} C_{F}}{\pi k_{\perp}^{2}}\left\{-\gamma(\xi / y) \frac{1+y^{2}}{(\bar{y})_{+}} \ln \frac{\mu^{2}}{k_{\perp}^{2}}-2 \gamma(\xi)\left(\frac{1}{\bar{y}}\right)_{+}\left(\ln \tilde{b}^{2} k_{\perp}^{2}+\ln \frac{y-\xi}{\xi \bar{\xi}^{2}}+\frac{1}{y} \ln \frac{\xi \bar{\xi}}{y}\right)\right. \\
& +\delta(\bar{y})\left[\gamma(\xi)\left(\ln \frac{\tilde{b}^{4} k_{\perp}^{4}}{\bar{\xi} \xi^{2}} \ln \bar{\xi}-\frac{1}{2} \ln ^{2}\left(\bar{\xi}^{2} \zeta_{u}^{2} \tilde{b}^{2} e^{-1}\right)-2 \operatorname{Li}_{2}\left(-\frac{\xi}{\bar{\xi}}\right)-\frac{3 \pi^{2}}{2}-\frac{7}{2}\right)+3 \bar{\xi}+1\right] \\
& +\left(\frac{2+\bar{\xi}^{2}}{\bar{\xi}}-\frac{\bar{y}}{\bar{\xi}^{2}}+1\right) \ln \frac{\bar{y} x}{\xi \bar{\xi}^{2}}+\frac{2(\bar{\xi}+1)(y-\xi)+4}{y} \ln \frac{\xi \bar{\xi}}{y \bar{y}}+\left(2 y-\xi-(y+1) \frac{x^{2}}{y^{2}}\right) \ln \frac{\xi x}{y^{2} \bar{y}} \\
& \left.+\frac{\gamma(\xi)}{\bar{\xi}^{2}}(\bar{\xi}+x) \ln \tilde{b}^{2} k_{\perp}^{2}+\frac{2 \gamma(\xi)}{y} \ln \bar{y}+\frac{3 \bar{y}+1}{\bar{\xi}}-\frac{2 \bar{y}}{\bar{\xi}^{2}}+2 \xi-\frac{\xi^{2}}{y^{2}}-\frac{\xi}{y}-y\right\}+\mathscr{O}\left(\alpha_{s}^{2}\right) .
\end{aligned}
$$

For the gluon initial state in Fig.[1], it is clear that the collinear divergence in figure Fig. B1(a) can be factorized into the quark-in-gluon PDF, and that in Fig. B](b) is factorized into the fragmentation function of a quark to a photon. The hard scattering coefficients are determined as:

$$
\begin{aligned}
& \hat{\mathscr{H}}_{g q}\left(x, \xi, z, b, k_{\perp}\right)=\frac{4 \pi \alpha_{s}}{k_{\perp}^{2}}\left(x^{2}+\bar{x}^{2}\right) \bar{x} z^{2} \delta(\bar{x} z-\xi), \\
& \hat{\mathscr{H}}_{g}\left(x, \xi, b, k_{\perp}\right)=\frac{e^{2} \alpha_{s}}{2 \pi k_{\perp}^{2}}\left\{-\left(y^{2}+\bar{y}^{2}\right)\left[\left(\frac{x^{2}}{y^{2}}+1\right) \ln \frac{\xi x \mu^{2}}{\bar{y} y^{2} k_{\perp}^{2}}+\frac{\xi^{2}}{y^{2}}\right]+2\left(x^{2}+\bar{y}^{2}\right) \ln \frac{x \bar{y}}{\bar{x} y}\right. \\
& \left.\quad-\left(\bar{x}^{2}+x^{2}\right)\left(\frac{\bar{y}^{2}}{\bar{x}^{2}}+1\right) \ln \frac{\xi^{3} x \mu^{2}}{\bar{y} \bar{x}^{2} k_{\perp}^{2}}-2\left(x^{2}+\bar{y}^{2}-\bar{\xi}\right) \frac{\bar{\xi}-2 x \bar{y}}{\bar{x} y}+\xi\left(\frac{y}{\bar{x}}+\frac{\bar{x}}{y}\right)+8 x \bar{y}\right\} .
\end{aligned}
$$

From our result, we can also directly derive the evolution equations of $\mu$ and $\zeta_{u}$ as:

$$
\begin{aligned}
& \frac{\partial \hat{F}_{q}\left(x, b, \xi, k_{\perp}, \zeta_{u}, \mu\right)}{\partial \ln \mu}=2 \gamma_{F} \hat{F}_{q}\left(x, b, \xi, k_{\perp}, \zeta_{u}, \mu\right), \quad \gamma_{F}=\frac{3 \alpha_{s} C_{F}}{4 \pi}+\mathscr{O}\left(\alpha_{s}^{2}\right) \\
& \frac{\partial \hat{F}_{q}\left(x, b, \xi, k_{\perp}, \zeta, \mu\right)}{\partial \ln \zeta_{u}}=-\frac{\alpha_{s} C_{F}}{\pi}\left(\ln \frac{x^{2} \zeta_{u}^{2} b^{2} e^{2 \gamma_{E}-1}}{4}\right) \hat{F}_{q}\left(x, b, \xi, k_{\perp}, \zeta_{u}, \mu\right)+\mathscr{O}\left(\alpha_{s}^{2}\right) .
\end{aligned}
$$

the evolution equation of $\zeta_{u}$ is called as Collins-Soper equation[[]], which is very useful for resummation of large log's in perturbation theory. As expected, these two equations are the same as those of quark TMD PDFs.

\subsection{Factorization of the integrated fracture function}

When the transverse momentum $k_{A \perp}$ is not observed, i.e., integrated out, we need the integrated fracture function. It can also be factorized further when $k_{\perp}$ is large. The factorization takes the 
same form as that for the TMD fracture function:

$$
F_{q}\left(x, \xi, k_{\perp}\right)=\sum_{a} \int \frac{d y}{y} f_{a / h}(y)\left[\mathscr{H}_{a}\left(x / y, \xi / y, k_{\perp}\right)+\sum_{b} \int \frac{d z}{z} D_{b}(z) \mathscr{H}_{a b}\left(x / y, \xi / y, z, k_{\perp}\right)\right] .
$$

In the calculation of the integrated fracture function, the gauge link is set along the light-cone direction, because there is no rapidity divergence in the final results. We refer to Ref.[[]] for the detailed calculations for each diagram and the results of the hard scattering coefficients. We emphasize that from the results, we can derive explicitly the renormalization scale $\mu$-evolution of the integrated fracture function. It is given by

$$
\frac{\partial F_{q}\left(x, \xi, k_{\perp}, \mu\right)}{\partial \ln \mu}=\frac{\alpha_{s}}{\pi} \int_{x}^{1-\xi} \frac{d y}{y} P_{q q}(x / y) F_{q}\left(y, \xi, k_{\perp}, \mu\right)+\cdots,
$$

where $P_{q q}(x)=C_{F}\left[\left(1+x^{2}\right) /(1-x)_{+}+\frac{3}{2} \delta(1-x)\right]$. The form of the evolution equation is the same as the standard quark collinear PDF. This is due to the factor that the integrated fracture function shares the same U.V. divergent subgraphs with the standard quark collinear PDF[四].

\section{Summary}

We showed that the hadronic tensor for the diffractive Drell-Yan lepton pair and photon production in hadron collisions can be factorized in terms of TMD or integrated fracture functions for different kinematic regions. Explicit one loop calculations show that both the TMD and integrated fracture functions can be further factorized into parton distribution and fragmentation functions when the transverse momentum is large or the impact parameter is small. Perturbative coefficients are extracted. In addition, the evolution equations for TMD and integrated fracture functions are derived. They take the same form as those for quark TMD and collinear PDFs respectively.

\section{References}

[1] L. Trentadue and G. Veneziano, Phys. Lett. B 323, 201 (1994). doi:10.1016/0370-2693(94)90292-5

[2] X. P. Chai, K. B. Chen, J. P. Ma and X. B. Tong, arXiv:1903.00809 [hep-ph].

[3] J.C. Collins, D.E. Soper and G. Sterman, Nucl. Phys. B250 (1985) 199, Nucl. Phys. B261, 104 (1985).

[4] A. Berera and D. E. Soper, Phys. Rev. D 53, 6162 (1996) doi:10.1103/PhysRevD.53.6162 